\title{
Noise Analysis in Distributed Amplifiers with Feedback-Active Load
}

\author{
J. Vicente Terán ${ }^{1}, \mathrm{M}^{\mathrm{a}}$ Luisa de la Fuente ${ }^{1}$, Ernesto Limiti ${ }^{2}$, Beatriz Aja ${ }^{1}$, Eduardo Artal ${ }^{1}$ \\ ${ }^{1}$ Department of Communication Engineering, University of Cantabria \\ Plaza de la Ciencia s/n, Santander, 39005, Spain \\ *josevicente.teran@unican.es \\ 2 Electronics Engineering Dept., University of Roma "Tor Vergata" \\ Via del Politecnico, 1 - 00133 Roma, Italy
}

\begin{abstract}
This paper presents the noise analysis of amplifiers when the input line termination is implemented via an active load. The noise figure of distributed amplifiers is analysed to determine the effect of the gate and drain terminations. The noise of a transistor configured as a one-port device has been analysed and a lower noise temperature has been achieved as compared to a conventional resistor. In order to improve the noise performance of the overall amplifier, a parallel feedback has been added to the active load, and its effects are analysed in terms of noise. A distributed amplifier has been designed as a test vehicle of the analysis and characterized with a resistor as input line termination and with an active load to demonstrate the improvement in noise figure at frequencies below $2 \mathrm{GHz}$. The resulting amplifier exhibits $10.9 \mathrm{~dB}$ of small signal gain from 1 to $5 \mathrm{GHz}$, with a reduction of $0.6 \mathrm{~dB}$ in noise figure below $2 \mathrm{GHz}$ when the input line termination is replaced by an active load.
\end{abstract}

\section{Introduction}

Distributed amplifiers (DAs) are usually designed to provide flat gain response over wide bandwidths. Resulting noise performance is typically poor, especially at low frequencies [1]-[3], where the noise introduced by the line termination dominates the noise of the active devices composing the amplifier. As long as the frequency increases, the noise of the active devices begins to be more important than the line termination contribution. One possible technique to minimize noise involves a change in the temperature of line termination. Previous works have adopted active loads as line terminations in order to improve the noise performance [4]-[6], although only simulation results have been presented and the influence of the active load in the overall amplifier, in terms of noise, is not completely explained. In [7]-[8], active load terminations are used in order to extend the low-frequency gain, but noise performance is not evaluated.

This work provides an extra overview over the noise reduction in a distributed amplifier when using feedback-active loads as line terminations and a mathematical analysis is proposed to explain such noise reduction phenomena. A small-signal analysis of a feedback transistor including noise sources demonstrates the resulting lower noise temperature of such arrangement, as compared to a conventional resistive load. Moreover, the effect of the temperature in the line loads of the distributed amplifier is discussed and the major contributors to the noise temperature are underlined. 
Based on the mathematical analysis, a simple two-stage distributed amplifier is designed in two configurations, namely a reference resistively-terminated one and an actively terminated amplifier as a test vehicle of the study. The frequency band of the amplifier spans from 1 to $5 \mathrm{GHz}$.

Finally, fabrication and measured results are presented for both amplifiers, demonstrating the improvement achieved in terms of noise performance when an active terminating load is used.

\section{Noise Figure}

In this section, the noise figure of a conventional distributed amplifier is analysed. Noise contributions of drain and gate line terminations are evaluated. Then the noise temperature of an active load considered as a one port device and implemented with a feedback transistor, is obtained.

\section{A. Noise figure of distributed amplifiers}

Noise in distributed amplifiers has been previously discussed by several authors [1], [2]. The noise factor of the amplifier is defined as

$$
F=1+\frac{N_{a}}{k \cdot T_{o} \cdot B \cdot G_{f}}
$$

where $N_{a}$ is the added noise from the amplifier to its output, $G_{f}$ is the distributed amplifier forward gain from the input to the output, $k$ is Boltzmann's constant, $T_{o}$ is $290 \mathrm{~K}$ and $B$ is the bandwidth where noise is measured ( $1 \mathrm{~Hz}$ will be assumed). Added noise can be decomposed in three terms as follows,

$$
N_{a}=N\left(Z_{d}\right)+N\left(Z_{g}\right)+N_{F E T}
$$

where $N\left(Z_{d}\right)$ is the noise power contribution of the drain line termination $Z_{d}$ and $N\left(Z_{g}\right)$ is the noise power contribution of the gate line termination $Z_{g}$. $N_{F E T}$ is the overall noise associated to the transistors composing the amplifier. The contributions of the line loads are defined as,

$$
\begin{array}{r}
N\left(Z_{d}\right)=k \cdot T_{o} \cdot B \\
N\left(Z_{g}\right)=k \cdot T_{o} \cdot B \cdot G_{r}
\end{array}
$$

where $G_{r}$ is the gain of the distributed amplifier in reverse configuration, which corresponds to the available gain from the input to the drain line termination.

Taking into account expressions (2), (3), and (4) and assuming noiseless transistors $\left(N_{F E T}=0\right)$, the noise factor due to contributions from line terminations only can be rewritten as

$$
F=1+\frac{1}{G_{f}}+\frac{G_{r}}{G_{f}}
$$


where the forward and reverse gains of the amplifier are explicitly indicated [1] in (6) and (7) under the assumption that drain and gate transmission lines exhibit the same phase constant and are lossless.

$$
\begin{gathered}
G_{f}=\frac{n^{2} \cdot g_{m}^{2} \cdot Z_{g} \cdot Z_{d}}{4} \\
G_{r}=\frac{g_{m}^{2} \cdot Z_{g} \cdot Z_{d}}{4} \cdot\left(\frac{\sin n \beta}{\sin \beta}\right)^{2}
\end{gathered}
$$

As already defined, $Z_{g}$ and $Z_{d}$ are gate and drain lines terminating impedances respectively (usually $50 \Omega$ ), $n$ is the number of stages of the DA (number of transistors), $\beta$ is the phase propagation constant of the transmission line, and $g_{m}$ is the transconductance of each transistor (identical transistors are assumed). The function $(\sin n \beta / \sin \beta)^{2}$ can be approximated by $n^{2}$ when $n \beta$ is close to 0 or $\pi$. In this case $G_{f}=G_{r}$.

In order to improve noise performance, the impact of the temperatures of the loads in the global noise figure of the amplifier is analysed. If a temperature $T_{g}\left(\neq T_{o}\right)$ is assumed in gate line termination keeping $T_{d}=T_{o}$ in drain line termination, its noise contribution $N\left(Z_{g}\right)$ is

$$
N\left(Z_{g}\right)=k \cdot T_{g} \cdot B \cdot G_{r}
$$

The resulting amplifier noise factor, $\mathrm{F}$, from (5), is,

$$
F=1+\frac{1}{G_{f}}+\frac{G_{r}}{G_{f}} \cdot \frac{T_{g}}{T_{o}}
$$

On the other hand, if a temperature $T_{d}\left(\neq T_{o}\right)$ is assumed for the drain line termination keeping $T_{g}=T_{o}$ in gate line termination, its noise contribution $N\left(Z_{d}\right)$ is,

$$
N\left(Z_{d}\right)=k \cdot T_{d} \cdot B
$$

The noise factor of the distributed amplifier in (5) results in (11).

$$
F=1+\frac{T_{d}}{T_{o}} \cdot \frac{1}{G_{f}}+\frac{G_{r}}{G_{f}}
$$

The effect in the overall noise figure of a temperature change in line loads can be evaluated separately, considering expressions (9) and (11). Fig. 1 shows how the noise figure $(10 \cdot \log (\mathrm{F}))$ changes with each load temperature when the other is kept constant for a distributed amplifier with $G_{f}=G_{r}=10 \mathrm{~dB}$. The impact of the gate line termination temperature is significantly higher if compared to the drain one as it can be expected since $T_{g}$ only is affected by the amplifier forward gain. From this result, it can be concluded that temperature changes in drain line termination will produce a negligible effect in the amplifier noise figure. 


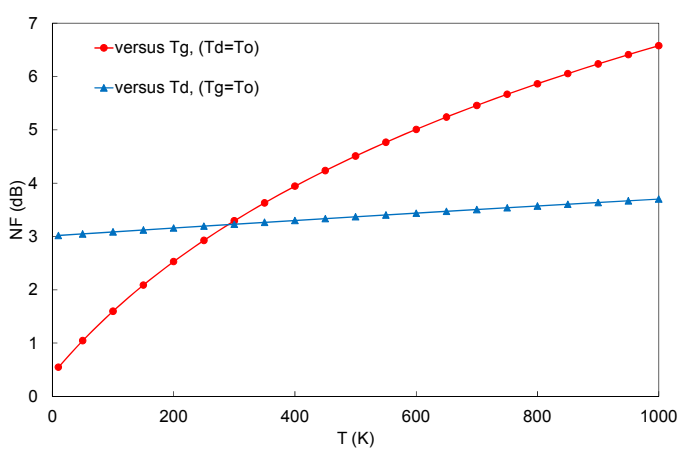

Fig. 1. Noise figure of a distributed amplifier in (9) and (11) versus line termination temperature.

\section{B. Noise temperature of an active load}

An active load can be implemented using a feedback transistor [9]-[11]. The noise temperature of such active load can be analysed through a simplified small-signal model (Fig. 2) of the high electron mobility transistor (HEMT).

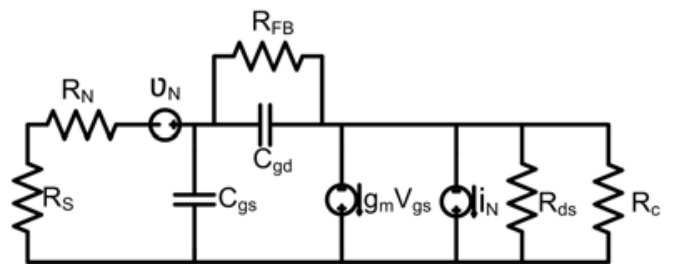

Fig. 2. Small signal model for noise temperature analysis in a HEMT.

Noise sources $v_{N}$ and $i_{N}$ can be obtained from transistor noise parameters (measured or provided by device manufacturer). A possible noise model for the transistor is suggested by Pucel et al. [12]. On the other hand, those noise voltage source and noise current source can be expressed by the equivalent noise resistance $R_{n}$ and equivalent noise conductance $G_{n}$, which are defined in [13], as (12)-(13), where T is the physical temperature.

$$
\begin{aligned}
& \overline{v_{N}^{2}}=4 \cdot k \cdot T \cdot B \cdot R_{n} \\
& \overline{l_{N}^{2}}=4 \cdot k \cdot T \cdot B \cdot G_{n}
\end{aligned}
$$

Applying superposition in the circuit of Fig. 2, noise currents can be obtained. The RMS value for the input current, $i_{i}$, due to drain noise current source is.

$$
\overline{\iota_{l}^{2}}=\left|-i_{N} \cdot H_{i}\right|^{2}=\bar{l}_{N}^{2} \cdot\left|H_{i}\right|^{2}
$$

where the transfer function $H_{i}$ is, 


$$
\left|H_{i}\right|^{2}=\left|\frac{Z_{d s} \cdot Z_{g s}}{\left(Z_{g s}+R_{s}+R_{n}\right) \cdot\left(Z_{d s}+\frac{R_{F B} \cdot Z_{g d}}{R_{F B}+Z_{g d}}\right)+\left(R_{s}+R_{n}\right) \cdot Z_{g s}+g_{m} \cdot\left(R_{s}+R_{n}\right) \cdot Z_{d s} \cdot Z_{g s}}\right|^{2}
$$

$R_{F B}$ is the gate to drain feedback resistance. $Z_{d s}, Z_{g d}$, and $Z_{g s}$ are

$$
Z_{d s}=R_{d s} / / R_{c} \quad Z_{g d}=\frac{1}{j \cdot \omega \cdot C_{g d}} \quad Z_{g s}=\frac{1}{j \cdot \omega \cdot C_{g s}}
$$

The RMS value for the input current, $i_{v}$, due to gate noise voltage source is

$$
\overline{\iota_{v}^{2}}=\left|v_{N} \cdot H_{v}\right|^{2}=\overline{v_{N}^{2}} \cdot\left|H_{v}\right|^{2}
$$

where the transfer function $H_{v}$ is,

$$
\left|H_{v}\right|^{2}=\left|\frac{1}{R_{s}+R_{n}}-\frac{1}{\left(R_{s}+R_{n}\right)+\frac{\left(R_{s}+R_{n}\right)^{2}}{Z_{g s}}+\left(R_{s}+R_{n}\right)^{2} \cdot\left(1+g_{m} \cdot Z_{d s}\right) \cdot \frac{1}{Z_{d s}+\frac{R_{F B} \cdot Z_{g d}}{R_{F B}+Z_{g d}}}}\right|^{2}
$$

The noise current at the input impedance $\left(R_{S}\right)$ is:

$$
\overline{l_{T}^{2}}=\overline{l_{N}^{2}} \cdot\left|H_{i}\right|^{2}+\overline{v_{N}^{2}} \cdot\left|H_{v}\right|^{2}
$$

The noise temperature can be computed at the input of the transistor $\left(R_{S}\right)$ as

$$
T_{n}=\bar{\iota}_{T}^{2} \cdot \frac{R_{S}}{k \cdot B}
$$

Considering (20) it is clear than the lower the noise current, the lower the noise temperature. Taking the small-signal model parameters (Fig. 2) for the HEMT transistor used in the amplifier design (more details in section 3), the effect of feedback can be evaluated. The values for the small signal parameters of the enhancement-mode pHEMT transistor ATF551M4 provided by AVAGO Technologies manufacturer, at the frequency of $3 \mathrm{GHz}$, are summarized in Table 1.

Table 1 Small signal parameters at $3 \mathrm{GHz}$ for the ATF551M4 HEMT transistor from AVAGO Technologies

$$
\begin{array}{ccccccc}
\mathrm{R}_{\mathrm{ds}}(\Omega) & \mathrm{R}_{\mathrm{c}}(\Omega) & \mathrm{R}_{\mathrm{n}}(\Omega) & \mathrm{R}_{\mathrm{s}}(\Omega) & \mathrm{C}_{\mathrm{gd}}(\mathrm{pF}) & \mathrm{C}_{\mathrm{gs}}(\mathrm{pF}) & \mathrm{gm}(\mathrm{mS}) \\
\hline 3507 & 260 & 6.97 & 50 & 0.17 & 0.32 & 44
\end{array}
$$

The noise current contributions for each noise source, $i_{i}(14)$ and $i_{v}(17)$, versus the feedback resistance $\left(R_{F B}\right)$ at three representative frequencies in the band of interest are compared in Fig. 3(a). The noise voltage 
source contribution is clearly predominant over the noise current one. Therefore, the effect of feedback in the transfer function $H_{v}(18)$ only is analysed.

Fig. 3(b) shows the influence of the feedback resistance in the transfer function $H_{v}(18)$ at three representative frequencies in the band of interest. $H_{v}$ magnitude has been magnified by 1000 for easier discrimination. As frequency increases, the required value of the optimum feedback resistance becomes smaller. For example, at $3 \mathrm{GHz}$ the behaviour exhibit a minimum close to $200 \Omega$. Such value represents therefore the optimum feedback value to minimize noise temperature.

Despite the feedback effect in $H v$ is small, its influence in the overall noise temperature of the active load is important as depicted in Fig. 3(c).

In Fig. 3(d), equivalent noise temperature of the active load is plotted versus frequency for different feedback resistances. The active load noise performance is better without feedback only for frequencies below $1 \mathrm{GHz}$. As feedback resistance decreases (from 1000 to $120 \Omega$ ) the noise temperature improvement is remarkable at higher frequencies, and minor at lower ones. As a consequence, the feedback effect provides a much lower noise temperature than $300 \mathrm{~K}$ of a passive $50 \Omega$ termination.

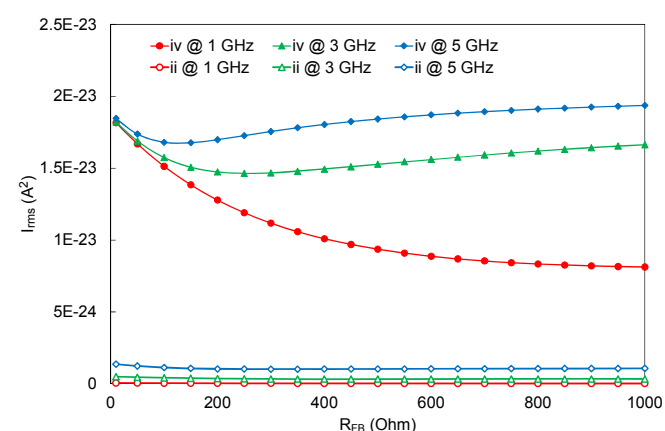

(a)

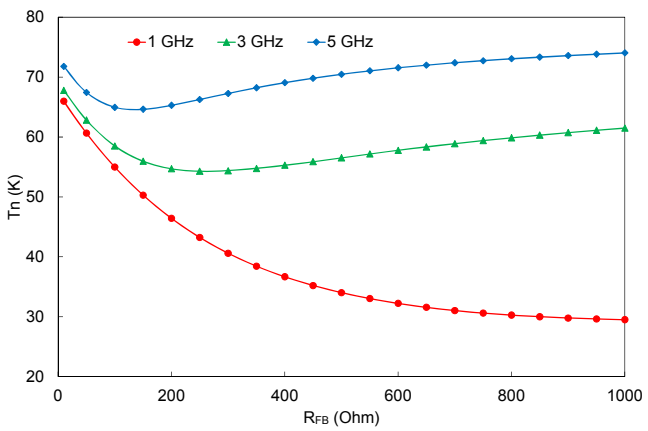

(c)

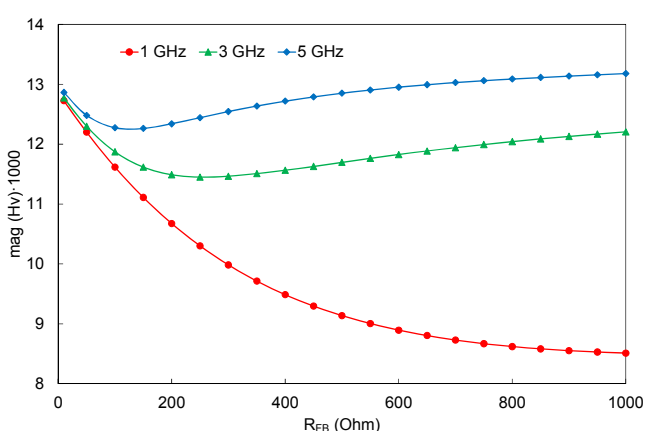

(b)

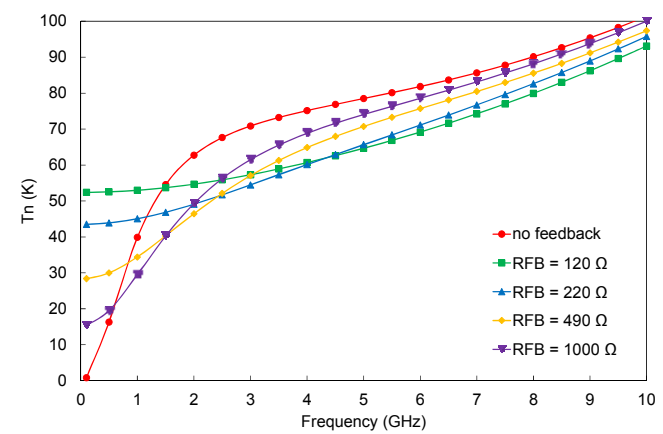

(d)

Fig. 3. (a) Noise current contributions for the active load due to noise sources versus feedback resistance $\left(R_{F B}\right)$ at three frequencies: noise voltage $i_{v}$ and current $i_{i}$ source contributions. (b) Magnitude of transfer function due to noise voltage source versus feedback resistance $\left(R_{F B}\right)$ at three frequencies. Overall noise temperature of the active load (c) versus feedback impedance at three frequencies, (d) versus frequency for different feedback impedances. 
The analysis developed in this section demonstrates that the active load represents an excellent solution to minimize low-frequency noise in a distributed amplifier. Introduction of feedback in the active load improves its noise performance in medium-high frequencies while providing a trade-off with a terminating impedance close to $50 \Omega$, necessary for a good distributed amplifier behaviour. A lower temperature in gate termination is more important in terms of noise figure than in the drain one, as is demonstrated in section 2.A. The active load will therefore be adopted as gate line termination.

\section{Design Example}

\section{A. Two-stage distributed amplifier with resistive terminations}

In order to validate the above analysis, a simple two-stage distributed amplifier is designed. Both gate and drain lines are terminated with $50 \Omega$ loads. The transistor is the same as the one used in previous section 2.B. The selected bias for each transistor is $v_{d s}=3 \mathrm{~V}, i_{d}=10 \mathrm{~mA}$. The transconductance for such bias point is $g_{m}=44 \mathrm{mS}$. Fig. 4 shows the electrical schematic of the amplifier. Passive elements have been implemented using lumped components.

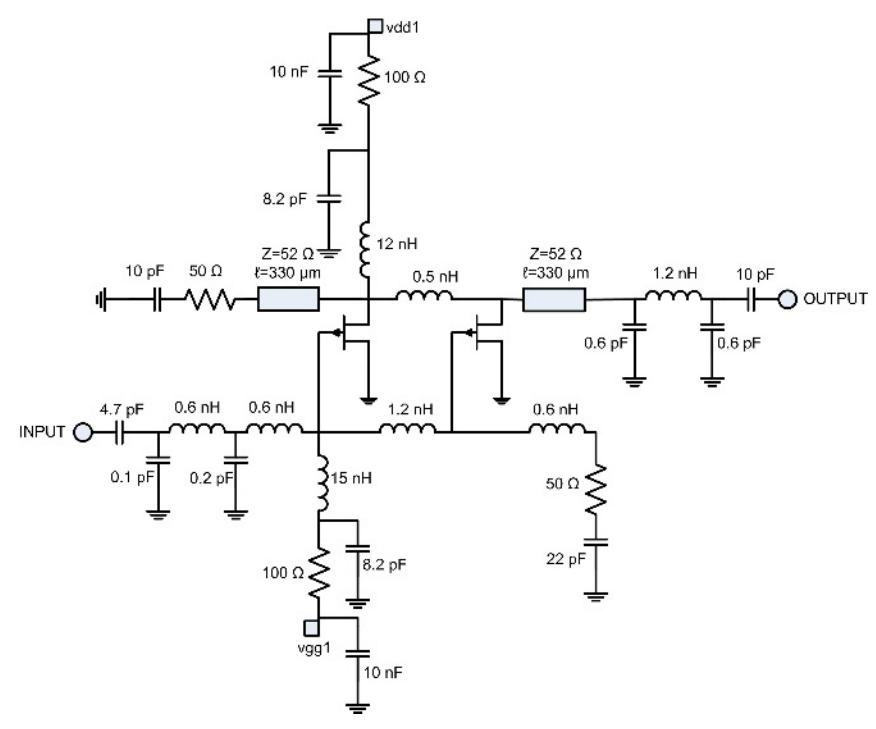

Fig. 4. Two cell distributed amplifier.

Table 2 Simulation and measurement results for distributed amplifier, DA, with resistive and active gate line termination. Mean values from 1 to $5 \mathrm{GHz}$

\begin{tabular}{|c|c|c|c|c|c|c|c|c|}
\hline & $\begin{array}{c}\text { Mean } \mathrm{S}_{21} \\
(\mathrm{~dB})\end{array}$ & $\begin{array}{c}\text { Slope } S_{21} \\
(\mathrm{~dB})\end{array}$ & $\begin{array}{c}\operatorname{Max}, \mathrm{S}_{11} \\
(\mathrm{~dB})\end{array}$ & $\begin{array}{c}\operatorname{Max} . \mathrm{S}_{22} \\
(\mathrm{~dB})\end{array}$ & $\begin{array}{c}\text { Mean NF } \\
(\mathrm{dB})\end{array}$ & $\begin{array}{c}\text { Max. NF } \\
(\mathrm{dB})\end{array}$ & $\begin{array}{l}\text { Min. NF } \\
(\mathrm{dB})\end{array}$ & $\begin{array}{c}\text { Slope NF } \\
\text { (dB) }\end{array}$ \\
\hline Simulation $-50 \mathrm{Ohm}$ line termination & 11.4 & 5.9 & -6.8 & -5.7 & 2.8 & 3.7 & 2.5 & 1.2 \\
\hline Measurements -50 Ohm line termination & 11.1 & 3.1 & -5.4 & -11.1 & 2.4 & 3.5 & 1.6 & 1.9 \\
\hline Simulation - active gate line termination & 11.7 & 4.8 & -6.3 & -6.4 & 2.1 & 2.4 & 1.6 & 0.8 \\
\hline Measurements - active gate line termination & 10.9 & 2.5 & -6.5 & -10.4 & 2.1 & 2.8 & 1.5 & 1.3 \\
\hline
\end{tabular}


$\Pi$ networks at the amplifier's input and output were designed to improve return loss. Part of the drain line is made up of microstrip lines in order to get the necessary inductive behaviour. Scattering parameters and noise simulation mean results in the 1-5 GHz frequency band are summarized in the first row of Table 2.

The effect of gate and drain line terminations is analysed in scattering and noise performance. Load resistances, $R_{g}$ and $R_{d}$, are varied separately from 10 up to $100 \Omega$. Fig. 5 shows the Scattering and noise figure performance when the gate load is swept keeping the drain load fixed to $50 \Omega$. There is a loss of flatness for small gate load resistance at $1 \mathrm{GHz}$, see Fig. 5(a). Moreover, large resistances are better in terms of noise (see Fig. 5(c)) below $2 \mathrm{GHz}$, but the effect is the opposite for higher frequencies.

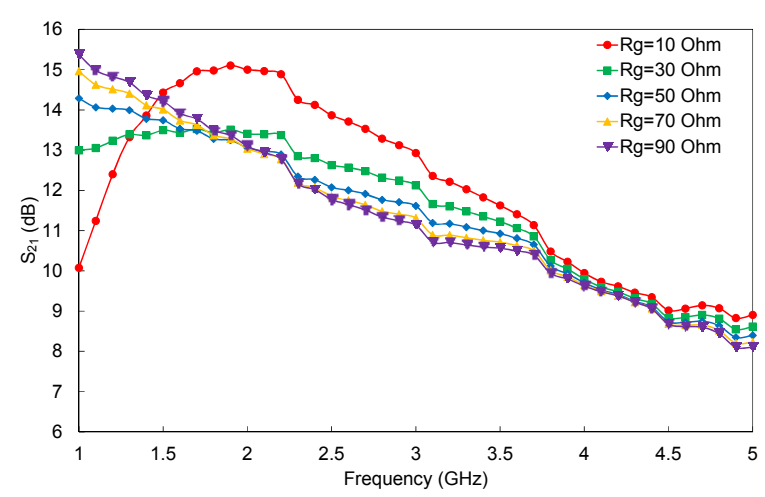

(a)

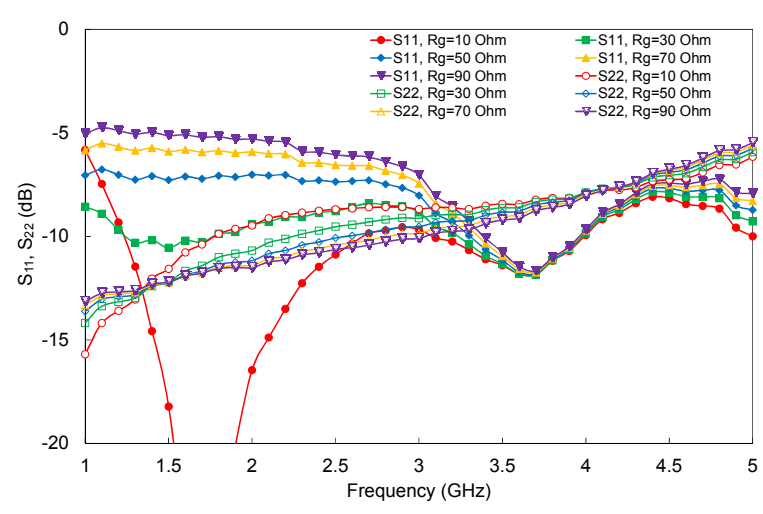

(b)

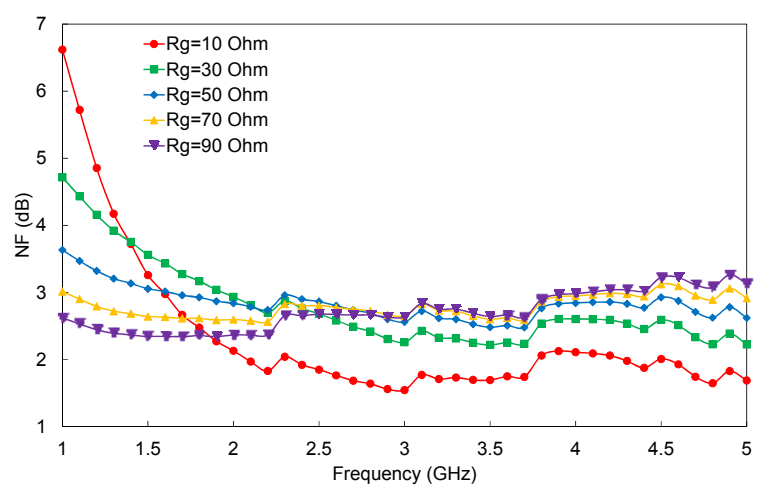

(c)

Fig. 5. Simulated Scattering parameters versus frequency and gate load for the distributed amplifier with resistive line terminations. Drain load $50 \Omega$. (a) $S_{21}$; (b) $S_{11}$ and $S_{22}$. (c) Simulated noise figure versus frequency and gate load for the distributed amplifier with resistive line terminations.

Fig. 6 shows the Scattering and noise figure performance when the drain load is swept keeping gate load fixed to $50 \Omega$. Very low drain load resistance does not allow good results in terms of gain, Fig. 6(a), and output matching, Fig. 6(b). Noise figure increases at high frequencies for small drain load resistances (see Fig. 6(c)). 


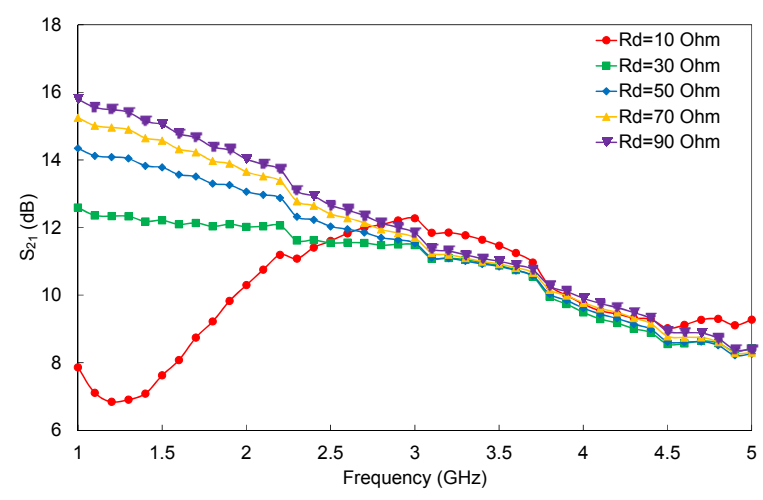

(a)

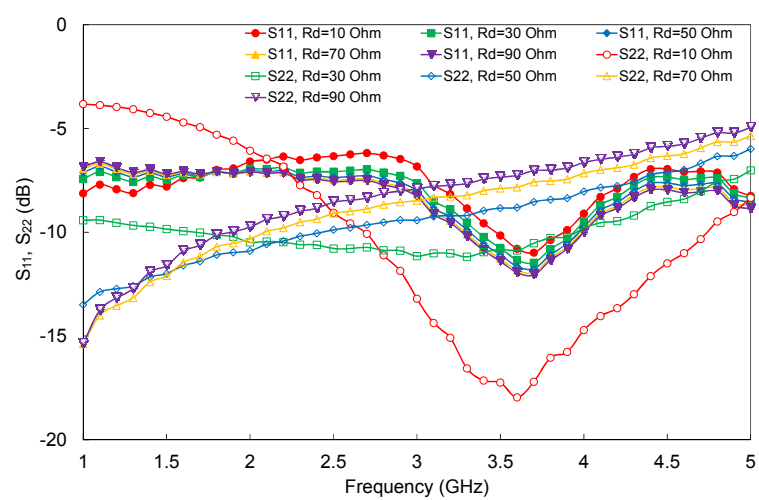

(b)

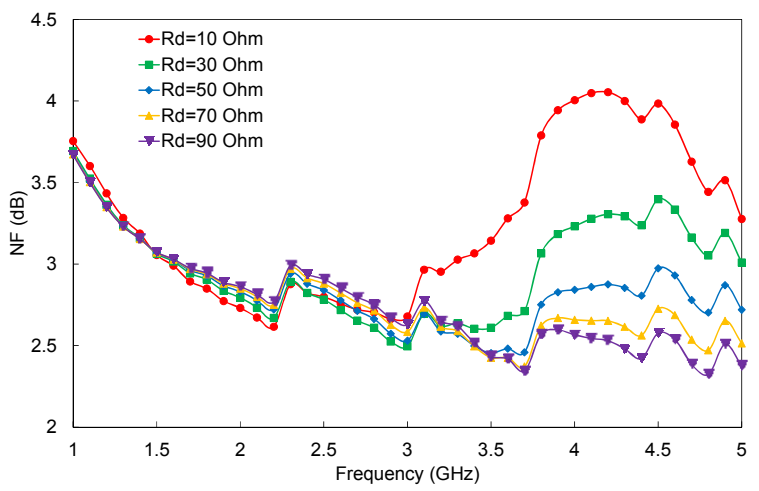

(c)

Fig. 6. Simulated Scattering parameters versus frequency and drain load for the distributed amplifier with resistive line terminations. Gate load 50 $\Omega$. (a) S21; (b) S11 and S22. (c) Simulated noise figure versus frequency and drain load for the distributed amplifier with resistive line terminations.

As a consequence, there is a noticeable effect in noise performance when terminating impedances are changed. For gate termination impedances lower than $50 \Omega$, noise figure is lower at high frequencies, but higher at low frequencies. A $50 \Omega$ impedance in gate termination is the best trade-off between noise figure and Scattering parameters in the whole frequency range. Regarding variations in drain termination, higher impedances provide a lower noise figure at high frequencies, but there is no influence at low frequencies.

\section{B. Active load}

In order to reduce the noise temperature of the loads of the distributed amplifier, active loads have been used taking into account the results obtained in section 2.B. A simple active load is implemented using a feedback transistor (the one used in section 2.B). The selected bias point is $v_{d s}=3 \mathrm{~V}$, and $i_{d}=649 \mu \mathrm{A}$. The transistor configuration shown in Fig. 7(a) without feedback is adopted. Noise power and temperature are obtained through expressions (21) and (22), where $v_{n}$ is the simulated noise voltage and $R_{i n}$ the simulated input resistance for the transistor. Fig. 7(b) shows a schematic of the active load with feedback resistor. 


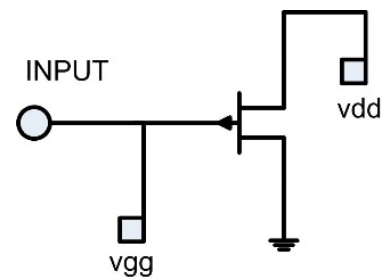

(a)

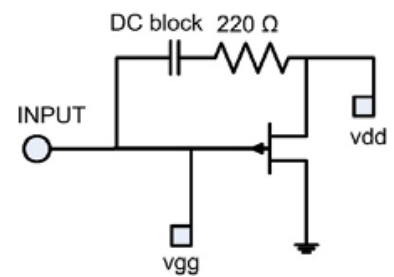

(b)

Fig. 7. (a) Schematic of a transistor, (a) common source, (b) common source with gate to drain feedback.

$$
\begin{gathered}
P_{n}=\frac{v_{n}^{2}}{4 \cdot R_{\text {in }}} \\
T_{N}=\frac{P_{n}}{k \cdot B}
\end{gathered}
$$

The noise temperature presented at the input of the transistor, and the simulated noise temperature of the common source transistor with gate to drain feedback are compared with that introduced by a $50 \Omega$ resistor load in Fig. 8(a).
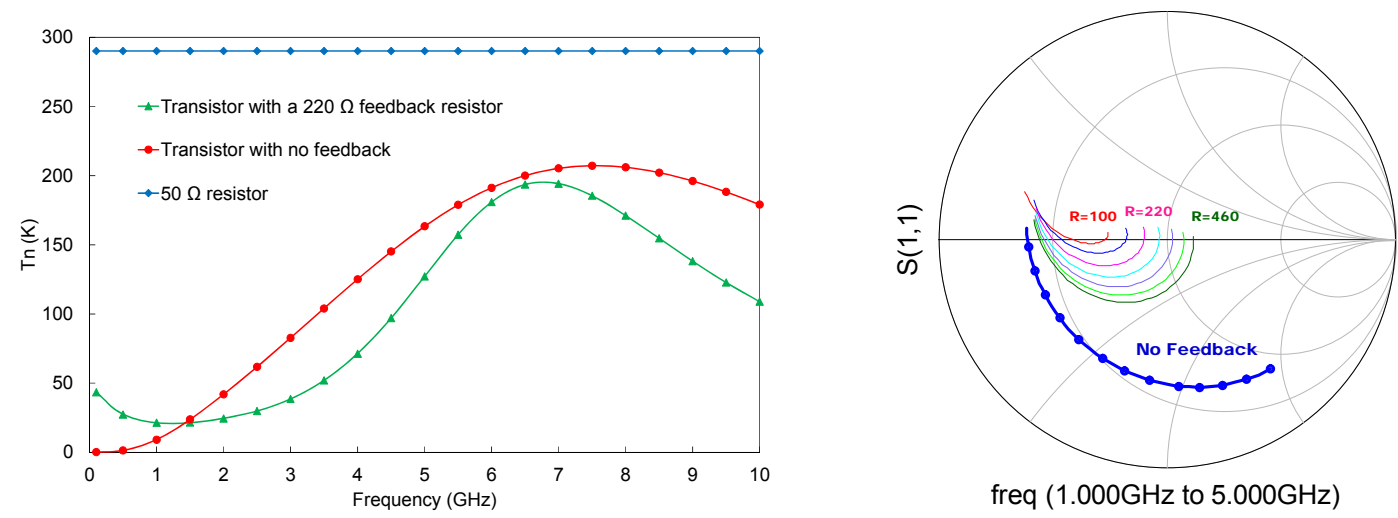

Fig. 8. (a) Noise temperature versus frequency for a $50 \Omega$ resistor and the transistor, with no feedback and with a $220 \Omega$ feedback resistor. (b) Input reflection coefficient of the active load without feedback and for different feedback resistances from 100 to $460 \Omega$, in steps of $60 \Omega$.

As obtained in section 2.B, noise temperature in a feedback transistor is strongly dependent on frequency and feedback resistance. Since a higher feedback resistance is better in terms of noise (section 2.B), a $220 \Omega$ was selected as the optimum value for central frequency in the band of interest, $3 \mathrm{GHz}$.

The improvement in noise temperature when the transistor is feedback evidences the need to the replace the resistive gate line termination by an active one in the amplifier. Moreover, the impedance of the active load remains close to $50 \Omega$ thanks to the feedback (Fig. 8(b)) mainly at low frequencies, improving both linear and noise performance, as shown in previous section 3.A. The influence in the amplifier noise 
of the active load will be mainly due to its noise temperature and the real part of its impedance, since its imaginary part (capacitive or inductive) has a negligible effect.

\section{Distributed amplifier with active gate line termination}

The active load previously designed is hence used as gate line termination of the DA. Since the improvement in noise performance due to a drain active load is small (section 2.A), no active load is considered for drain line avoiding the resulting increase in complexity.

Full schematic of the DA is shown in Fig. 9. Feedback resistor was adjusted to $150 \Omega$ to account for the influence of parasitic resistance of DC blocks and connection pads. Moreover, a bias network for the active load was implemented through a $2.7 \mathrm{k} \Omega$ resistor.

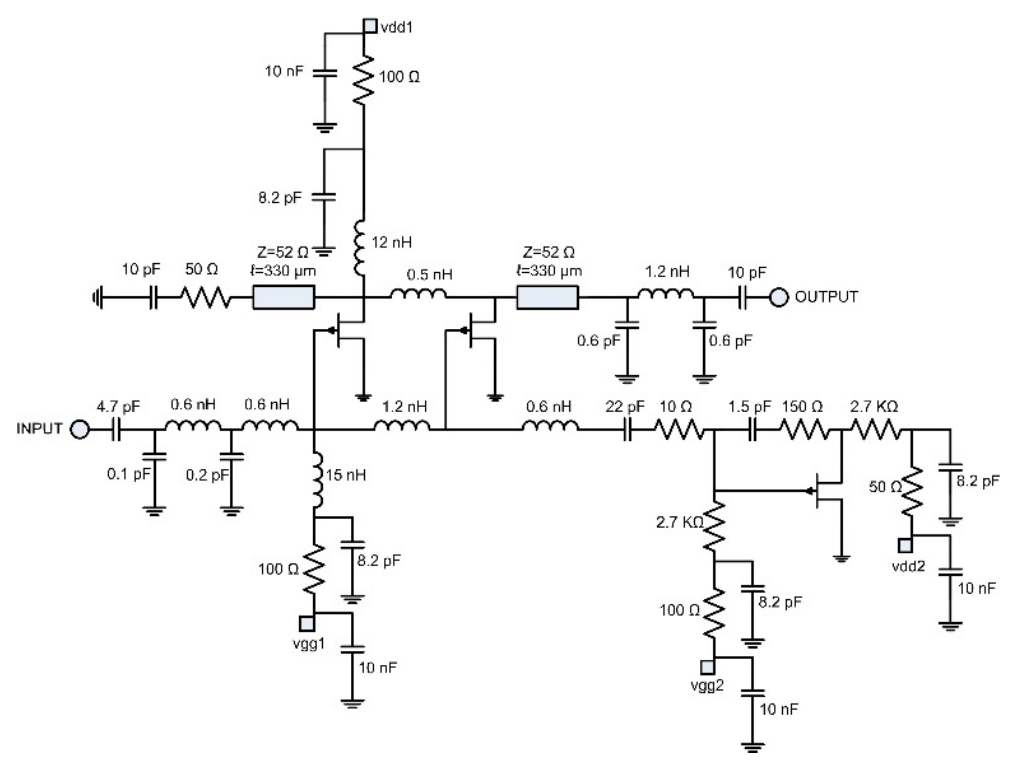

Fig. 9. Schematic of DA with active gate line termination.

Simulated results of the new configuration for the DA in the frequency band of interest, 1 to $5 \mathrm{GHz}$, are summarized in the third row of Table 2. The linear performance is similar to the one obtained with the traditional DA, but there is $1 \mathrm{~dB}$ improvement in noise figure performance. Mean noise figure is $2.1 \mathrm{~dB}$ and the slope between the maximum and the minimum noise figure in the frequency band of interest is $0.8 \mathrm{~dB}$ only. The improvement is higher below $2 \mathrm{GHz}$ because the influence of the gate line termination noise temperature is higher at low frequencies, while the noise of the active devices becomes more significant at high frequencies. 


\section{Performance of the DA: Fabrication and measured results}

\section{A. DA with resistive line terminations}

All the components for the implementation of the distributed amplifier are mounted on a 30 mils substrate from Arlon, (CUCLAD 250, with dielectric constant 2.5). Capacitors and inductances are carefully selected, from ATC ceramics and Johanson Technology respectively.

Network analyser PNA E8364A from Keysight Technologies is used as linear measurement setup. Noise test setup includes a noise figure meter N8975A and a noise source N4002A, both from Keysight Technologies. Measurements are presented in Fig. 10, Scattering performance in Fig. 10(a) and noise performance in Fig. 10(b). The bias point for the transistors has been adjusted for better performance: $i_{d}=20 \mathrm{~mA}$ and, $v_{d s}=4.05 \mathrm{~V}$. Mean results are summarized in the second row of Table 2 in the frequency band from 1 to $5 \mathrm{GHz}$. The amplifier achieves an average gain $11.1 \mathrm{~dB}$ and a mean noise figure of $2.4 \mathrm{~dB}$.

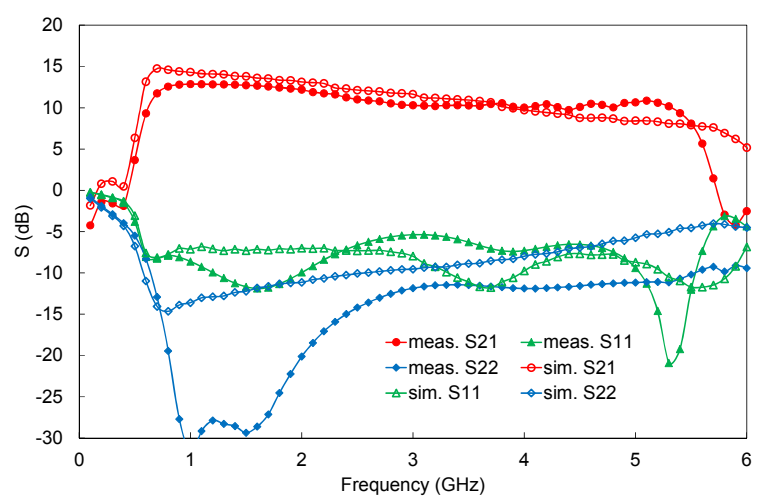

(a)

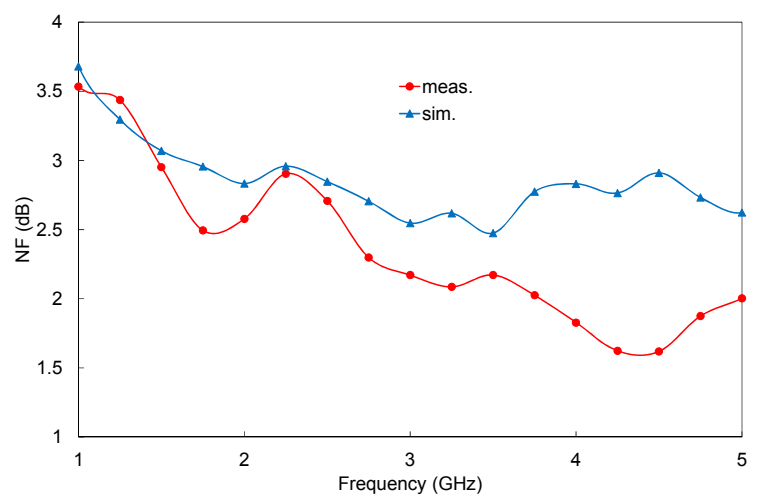

(b)

Fig. 10. (a) Measured and simulated Scattering parameters versus frequency for the DA with resistive line terminations, (b) measured and simulated noise figure (NF) versus frequency. 


\section{B. DA with active gate line termination}

The designed amplifier was reconfigured by replacing the resistive load termination with an active gate load. Fig. 11 shows the full manufactured DA with the active gate line termination. The bias point for the transistors in the two stages of the amplifier is $i_{d}=20 \mathrm{~mA}$ and, $v_{d s}=4.05 \mathrm{~V}$; the best bias point for the transistor for the active load is $v_{d s}=5.8 \mathrm{~V}$ and, $i_{d}=2 \mathrm{~mA}$. Both linear and noise performance of the amplifier are shown in Fig. 12.

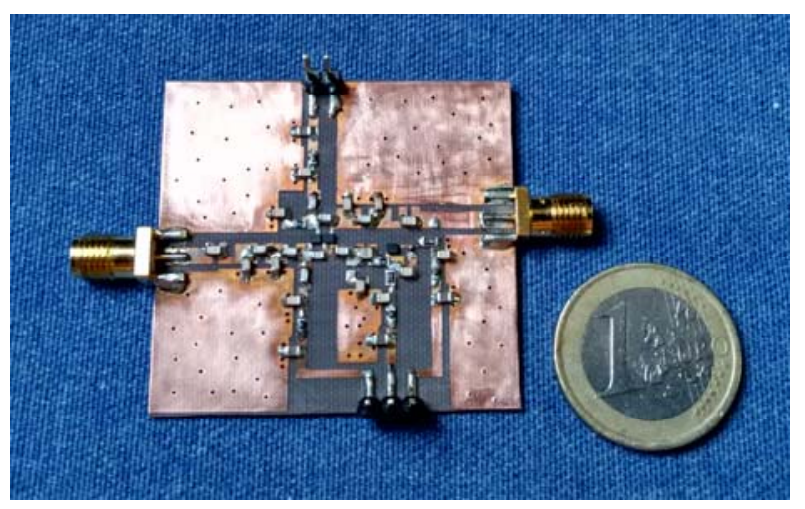

Fig. 11. Photo of the full DA with gate line active termination (Size $42.3 \times 44.7 \mathrm{~mm}^{2}$ ).

Mean results in the frequency band of interest, from 1 to $5 \mathrm{GHz}$, are summarized in the fourth row of Table 2. The amplifier exhibits an average gain of $10.9 \mathrm{~dB}$ and a mean noise figure of $2.1 \mathrm{~dB}$. Scattering performance does not change significantly (see Fig. 12(a)), but there is a mean reduction of $0.3 \mathrm{~dB}$ in terms of noise figure in the band (see Fig. 12(b)), and noise flatness is also improved. Fig. 12(c) shows a comparison between the noise figure of the DA with the active gate line termination and with resistive ones. The higher noise reduction is evident for frequencies below $2 \mathrm{GHz}$ where the improvement is $0.6 \mathrm{~dB}$. This result agrees with the simulation of the amplifier in section 3.C. This improvement at low frequency is clearly caused by the lower noise contribution of the active load, compared with the resistive one. On the other hand, the noise performance is nearly the same at higher frequencies when the mayor contribution to the total noise figure comes from the active devices who make up the amplifier. 


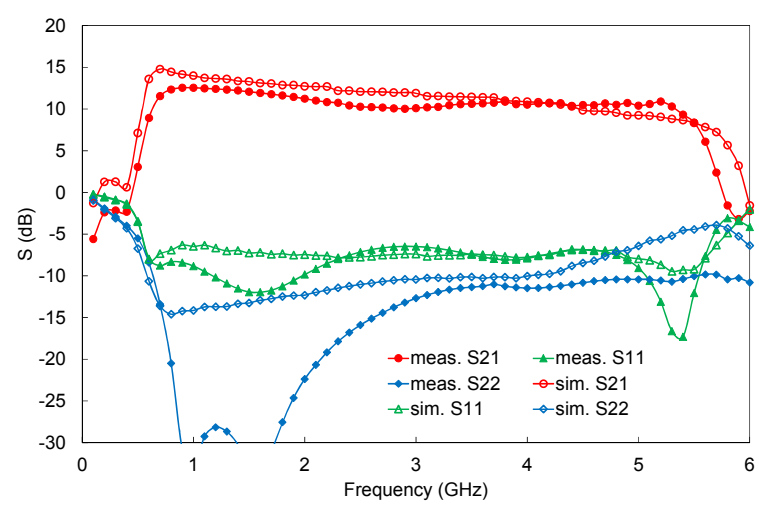

(a)

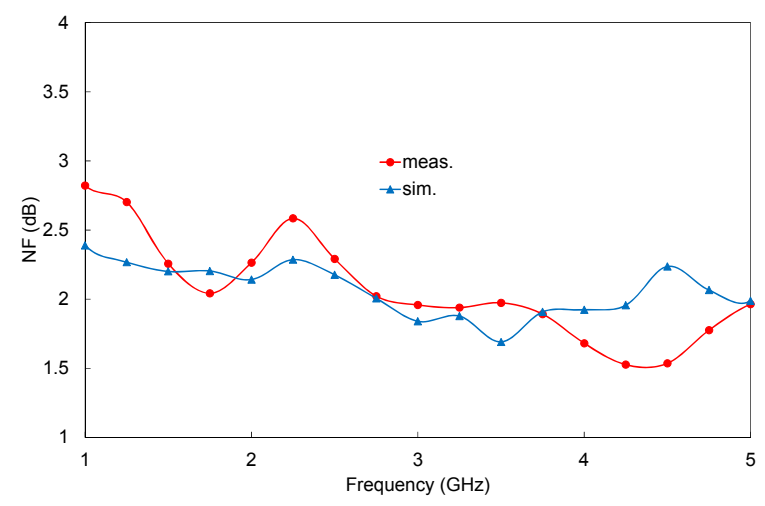

(b)

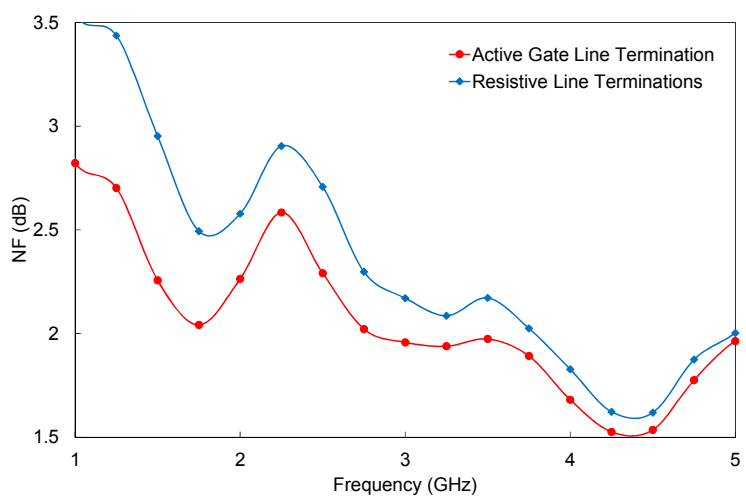

(c)

Fig. 12. (a) Measured and simulated Scattering parameters versus frequency for the distributed amplifier with active gate line termination, (b) measured and simulated noise figure (NF) versus frequency. (c) Comparison of Noise figure measurement of the DA versus frequency with the active gate line termination and with resistive ones.

\section{Conclusions}

A methodology for the improvement of noise performance in a DA at low frequencies has been investigated making use of an active feedback termination of the gate line. To this goal, the influence of temperature changes in DA line terminations has been analysed, demonstrating the major influence of gate line termination noise temperature as compared to the drain one. A mathematical formulation of noise temperature for an active load has been also presented. Noise figure expressions in a DA based on the active device small signal and noise parameters have been derived.

In order to verify the attained results, a hybrid two-stage distributed amplifier has been designed and fabricated both with resistive and active loads. Measured data result in broadband amplifier performance from 1 to $5.2 \mathrm{GHz}$ with $10.9 \mathrm{~dB}$ flat gain. A $0.6 \mathrm{~dB}$ noise figure improvement below $2 \mathrm{GHz}$ has been achieved when the active load is adopted as the gate line termination. 


\section{Acknowledgment}

The authors wish to thank Eva Cuerno for their tireless dedication and effort in assembling the circuit. Also the authors thank José Ángel García García for all contributed advices about technology during the design process.

This work was supported by the Ministerio de Economía y Competitividad from Spain under the CONSOLIDER-INGENIO 2010 program CSD2010-00064 reference, and the research program FPI BES2011-046199.

\section{References}

[1] Colin S. Aitchison: 'The Intrinsic Noise Figure of the MESFET Distributed Amplifier', IEEE Transactions on Microwave Theory and Techniques, 1985, 33, (6), pp. 460-466

[2] K. B. Niclas, "On Noise in distributed Amplifiers at Microwave Frequencies", IEEE Transactions on Microwave Theory and Techniques, 1983, 31, (8), pp. 661-668

[3] Kambiz Moez and Mohamed I. Elmasry, "A Low-Noise CMOS Distributed Amplifier for Ultra-Wide-Band Applications”, IEEE Transactions on Circuits and Systems, 2008, 55, (2), pp. 2076-2082

[4] P. K. Ikalainen, "Low-Noise Distributed Amplifier with Active Load", IEEE Microwave and Guided Wave Letters, 1996, 6, (1), pp. 7-9

[5] Kobayashi, K.W.; Denninghoff, D.; Miller, D. "A Novel 100 MHz-45 GHz GaN HEMT Low Noise Non-Gate-

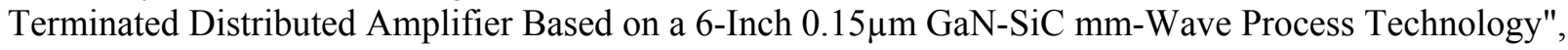
Compound Semiconductor Integrated Circuit Symposium (CSICS), 2015, pp. 1-4

[6] Shunji Kimura and Yuhki Imai, "0-40 GHz GaAs MESFET Distributed Baseband Amplifier IC's for HighSpeed Optical Transmission”, IEEE Transactions on Microwave Theory and Techniques, 1996, 44, (11), pp. 20762082

[7] K. W. Kobayashi, J. Cowles, L. T. Tran, A. Gutierrez-Aitken, T. R. Block, A. K. Oki, and D. C. Streit, "A 50MHz-55-GHz Multidecade InP-Based HBT Distributed Amplifier", IEEE Microwave and Guided Wave Letters, 1997, 7, (10), pp. 353-355

[8] Jinho Jeong and Youngwoo Kwon, "Monolithic Distributed Amplifier With Active Control Schemes for Optimum Gain and Group-Delar Flatness, Bandwidth, and Stability", IEEE Transactions on Microwave Theory and Techniques, 2004, 52, (4), pp. 1101-1110

[9] Buhles, P.M.; Lardizabal, S.M. "Design and characterization of MMIC active cold loads", Microwave Symposium Digest. 2000 IEEE MTT-S International, June 2000, 1, pp. 29-32

[10] K. B. Niclas, "Noise in Broad-Band GaAs MESFET Amplifiers with Parallel Feedback", IEEE Transactions on Microwave Theory and Techniques, 1982, 30, (1), pp. 63-70

[11] K. B. Niclas, "The Exact Noise Figure of Amplifiers with Parallel Feedback and Lossy Matching Circuits", IEEE Transactions on Microwave Theory and Techniques, 1982, 30, (5), pp. 832-835 
[12] Robert A. Pucel, Hermann A. Haus, and Hermann Statz: 'Signal and Noise Properties of Gallium Arsenide Microwave Field-Effect Transistors', Advances in Electronics and Electron Physics, 1975, 38, pp. 195-265

[13] H. Rothe, and W. Dahlke: 'Theory of Noisy Fourpoles', Proceedings if the IRE, 1956, 44, (6), pp. 811-818 